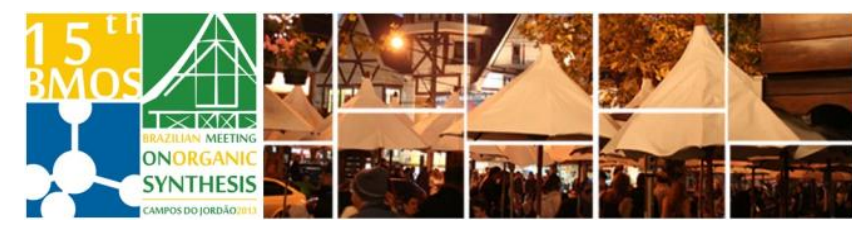

\title{
Study toward the convergent total synthesis of the aporphine alkaloid PO-3
}

\section{Allan F. C. Rossini and Cristiano Raminelli*}

Instituto de Ciências Ambientais, Químicas e Farmacêuticas, Universidade Federal de São Paulo, Diadema, SP, Brazil

*e-mail address: raminelli@unifesp.br

Keywords: benzyne chemistry, aporphine alkaloid, cycloaddition reaction

\section{INTRODUCTION}

Due to the great importance of benzyne as a highly reactive intermediate in organic chemistry, with applications in total syntheses ${ }^{1}$ and in preparations of functional materials, ${ }^{2}$ we intend to accomplish the convergent total synthesis of the aporphine alkaloid PO-3 (1), a natural dye of green color, ${ }^{3}$ employing as key reaction a [4+2] cycloaddition between 1-methylene-1,2,3,4-tetrahydroisoquinoline (3) and the benzyne derivative $\mathbf{7}$, formed from 2-(trimethylsilyl)aryl triflate (8), under mild reaction conditions.

\section{RESULTS AND DISCUSSION}

Attracted by the dye property of the aporphine alkaloid PO-3 (1), ${ }^{3}$ we decide to perform the total synthesis of the mentioned natural product of green color, employing a convergent synthetic route. Thus, according to the retrosynthetic analysis, the alkaloid PO-3 (1) can be obtained by functional group transformations from the intermediate 2 , which can be produced by the [4+2] cycloaddition reaction between 1-methylene-1,2,3,4-tetrahydroisoquinoline (3) and 2-(trimethylsilyl)aryl triflate (8), followed by a spontaneous dehydrogenation process ${ }^{3}$ (Scheme 1).

Scheme 1. Retrosynthetic analysis for the alkaloid PO-3 (1).

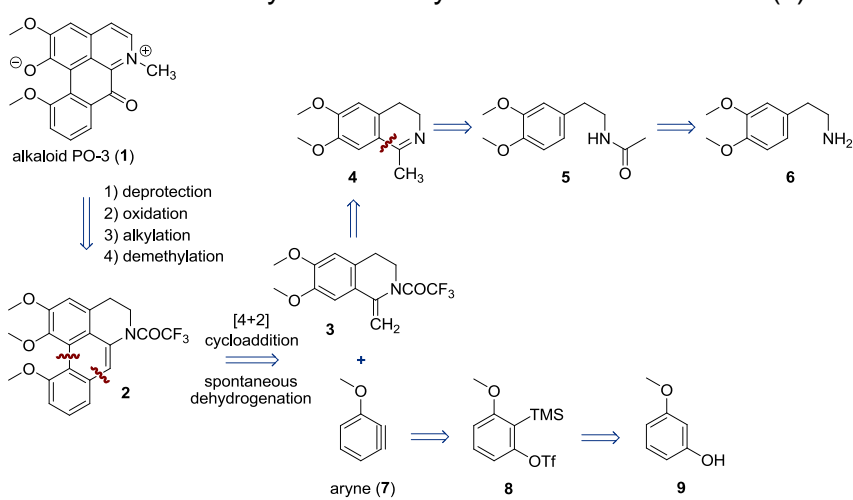

Guided by the retrosynthetic analysis outlined in Scheme 1, we started the synthesis to obtain the dye PO-3 (1), by the preparations of 1-methylene1,2,3,4-tetrahydroisoquinoline (3) (fragment $\mathbf{A}$ ) and of the silylaryl triflate $\mathbf{8}$ (fragment $\mathbf{B}$ ), which were obtained in good yields (Scheme 2).
Scheme 2. Synthetic route for the alkaloid PO-3 (1).

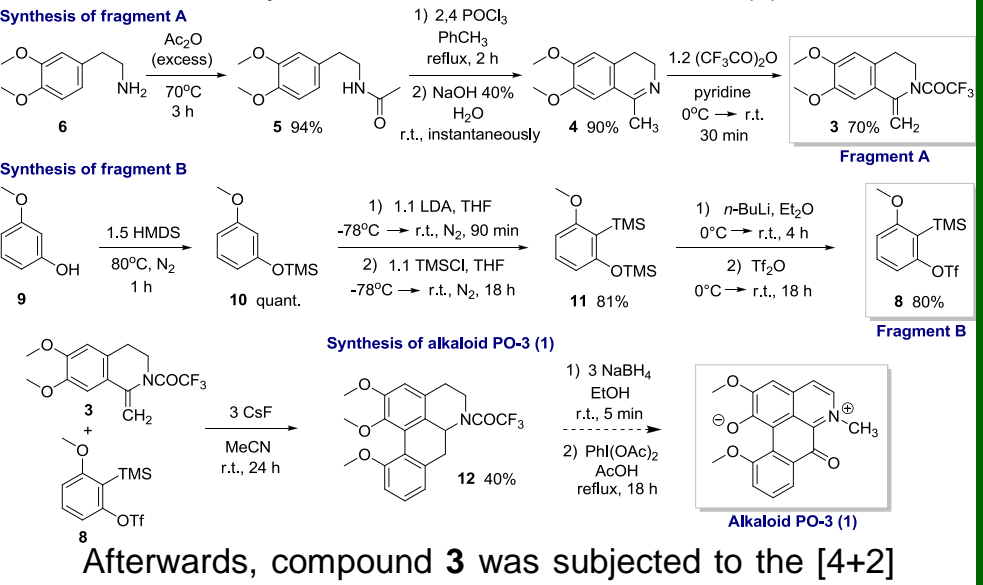

Afterwards, compound 3 was subjected to the [4+2] cycloaddition reaction with the aryne precursor $\mathbf{8}$, resulting in the highly regioselective formation of the intermediate 12 in $40 \%$ yield, which contains the basic structure of the alkaloid PO-3 (1). However, for our surprise, the process of spontaneous dehydrogenation did not occur. ${ }^{3}$ After optimizing the reaction which leads to the intermediate $\mathbf{1 2}$, we intend to obtain the alkaloid PO-3 (1) by reactions described in the literature ${ }^{3,4}$ (Scheme 2).

\section{CONCLUSION}

Fragments $\mathbf{A}$ and $\mathbf{B}$ were obtained by reactions that presented high yields. Compounds $\mathbf{3}$ and $\mathbf{8}$ are going to be used in the optimization of the [4+2] cycloaddition reaction, i.e., the key step for the convergent synthesis of the aporphine alkaloid PO-3 (1).

\section{ACKNOWLEDGEMENTS}

CNPq and FAPESP for financial support. CAPES for the scholarship of A.F.C.R.

\section{REFERENCES}

${ }^{1}$ (a) Tadross, P. M.; Stoltz, B. M. Chem. Rev. 2012, 112, 3550. (b) Gampe, C. M.; Carreira, E. M. Angew. Chem., Int. Ed. 2012, 51, 3766.

${ }^{2}$ (a) Lu, X.; Nikawa, H.; Tsuchiya, T.; Akasaka, T.; Toki, M.; Sawa, H.; Mizorogi, N.; Nagase, S. Angew. Chem., Int. Ed. 2010, 49, 594. (b) Shen, Y.-M.; Grampp, G.; Leesakul, N.; Hu, H.-W.; Xu, J.-H. Eur. J. Org. Chem. 2007, 3718.

${ }^{3}$ (a) Atanes, N.; Castelo, L.; Guitián, E.; Saá, C.; Saá, J. M.; Suau, R. J. Org. Chem. 1991, 56, 2984. (b) Saá, C.; Guitián, E.; Castedo, L.; Saá, J. M. Tetrahedron Lett. 1985, 26, 4559.

${ }^{4}$ Singh, O. V.; Huang, W.-J; Chen, C.-H.; Lee, S.-S. Tetrahedron Lett. 2007, 48, 8166. 